\title{
Besser als sein Ruf
}

\section{Das Pflegeneuordnungsgesetz aus der Perspektive eines Pflegeanbieters}

\author{
INGRID HASTEDT \\ Ingrid Hastedt ist Vorsitzende des \\ Vorstandes des Wohlfahrtswerks \\ für Baden-Württemberg. Die Stif- \\ tung des bürgerlichen Rechts ist ei- \\ ner der größten Träger der Altenhilfe \\ in Baden-Württemberg und betreut \\ mit 1.400 Mitarbeitern rund 2.000 \\ Menschen. Das Wohlfahrtswerk für \\ Baden-Württemberg ist auch Her- \\ ausgeber der im Jahre 1848 erstmals \\ erschienenen Fachzeitschrift Blätter \\ der Wohlfahrtspflege. \\ www.wohlfahrtswerk.de
}

\author{
Die neue Pflegereform bringt für pflegebedürftige \\ Menschen einige Verbesserungen. Auch für die \\ Dienste und Einrichtungen der Altenpflege eröffnen \\ sich neue Chancen zur Weiterentwicklung ihrer \\ fachlichen und wirtschaftlichen Konzepte.
}

Das am 30. Oktober 2012 in Kraft getretene Pflegeneuordnungsgesetz (PNG) wurde im Vorfeld heftig kritisiert. Zwei Aspekte haben diese Diskussion um die Reform der Pflegeversicherung dominiert: die Reichweite der Beitragseinnahmen je nach Beitragssatz und die Zutrittsschwelle zu Leistungen der Pflegekasse.

Das Pflegeneuordnungsgesetz erhöht den Beitragssatz für Arbeitnehmer nur um 0,1 Prozentpunkte und bleibt damit weit hinter der von vielen als notwendig erachteten Dimension zurück. Für die Praxis der Pflegeanbieter hat die Kaufkraft der betroffenen Menschen natürlich eine zentrale Bedeutung. Doch zum Tragen kommen Folgen einer heute zu geringen Anhebung des Beitragssatzes wegen der demografischen Entwicklung erst mittel- bis langfristig. Wie viel Pflege sich Menschen in zehn oder zwanzig Jahren werden leisten können, wenn der Beitragssatz - wie geschehen - nur marginal oder viel stärker angehoben worden wäre, stellt für Pflegeerbringer ein Thema weit in die Zukunft reichender strategischer Planungen dar.

Die Leistungserbringer bemerken dagegen bereits heute eine abnehmende Kaufkraft der Pflegekassenleistungen: Zwölf Jahre nach Einführung der Pflegeversicherung erhöhte die SGB XI-Reform von 2008 erstmals die Pflegesachund Pflegegeldleistungen für ambulante Leistungsbezieher. Dies geschah in drei Stufen zum 1. Juli 2008, zum 1. Januar 2010 und zum 1. Januar 2012.

Die nun Ende Oktober 2012 in Kraft getretene Pflegereform erwähnt dagegen keinerlei Überlegungen eines
Inflationsausgleichs. Für Pflegeheimbewohner heißt die seit 1996 eingefrorene Leistungshöhe, dass ihre aus der Pflegeversicherung gespeiste Kaufkraft faktisch parallel zum Ansteigen der Pflegesätze gesunken ist. Diese Situation führt zu einer wieder ansteigenden Zahl der Sozialhilfeempfänger in Pflegeheimen. Protest gegen diese schleichende Entwicklung und Forderungen nach einer an der Inflation oder Tarifentwicklung orientierten Leistungsanpassung gingen im Vorfeld der jüngsten SGB XI-Reform im Getöse anderer Themen unter.

Am lautesten wurde beklagt, dass das Pflegeneuordnungsgesetz keinen neuen Pflegebedürftigkeitsbegriff einführt, dass also $\mathbb{S} 14$ SGB XI unverändert bleibt. Das dort beschriebene Pflegebedürftigkeitsverständnis bildet durch Demenz ausgelösten Hilfebedarf nur unzureichend ab; Folgen somatischer Einschränkungen werden fokussiert.

Seit dem Jahre 2009 liegen Vorschläge des "Beirats zur Überprüfung des Pflegebedürftigkeitsbegriffs « zu einer Reform des Begriffs der Pflegebedürftigkeit vor, die diese Ungleichgewichte beseitigen würden und in der Fachwelt durchweg auf positive Resonanz stießen. Dass dieser Ansatz nach mehr als drei Jahren noch immer keinen Eingang in das SGB XI gefunden hat, begründet die tendenziell negative Rezeption des Pflegeneuordnungsgesetz in der Fachöffentlichkeit.

Doch wäre es falsch, dem Gesetzgeber vorzuwerfen, das Pflegeneuordnungsgesetz ließe die finanziellen 
Nöte pflegebedürftiger Menschen mit Demenz, die durch das Raster des $\mathbb{1 4}$ SGB XI fallen, außer Acht. Es begünstigt vor allem häuslich Betreute, bei denen kognitiv bedingte Einschränkungen der Alltagsbewältigung vorliegen, darunter auch solche ohne Pflegestufe. Was genau unter "erheblichem allgemeinem Unterstützungsbedarf im Alltag " verstanden wird, ist seit 2008 in $\$ 45$ a SGB XI definiert. Damals wurde mit $\mathbb{4} 45 \mathrm{~b}$ SGB XI ein monatlicher Betrag von 100 bzw. 200 Euro für nach $\mathbb{\$} 45$ a SGB XI Anspruchsberechtigte eingeführt. Das Pflegeneuordnungsgesetz führt für diese Personen ohne Pflegestufe, aber mit erheblich eingeschränkter Alltagskompetenz, monatliche Geldleistung (120 Euro) oder Sachleistung (bis zu 235 Euro) ein.

Wer die Anfänge der Pflegeversicherung miterlebt hat weiß, dass die Einführung eines an Pflegephänomenen orientierten Zugangs zu Pflegekassenleistungen erhebliche Umstellungen für die Pflegekräfte bedeutete. Und dass es mehrere Jahre dauerte, bis einstufungsrelevante Sachverhalte konsequent aus der Pflegedokumentation ableitbar waren und sich Routinen im Umgang mit unbefriedigendem Einstufungsverhalten von Gutachtern eingestellt hatten.

Positiv ist, dass der Praxis im Jahr 2013 erspart bleibt, sich in Windeseile auf die Anwendung neuer Einstufungskriterien einstellen zu müssen. Zu kurz liegen die im vergangenen Jahrzehnt eng getakteten Neuerungen für die Pflegeprofession zurück: Expertenstandards waren umzusetzen, ohne dass das System die für diese Organisations-

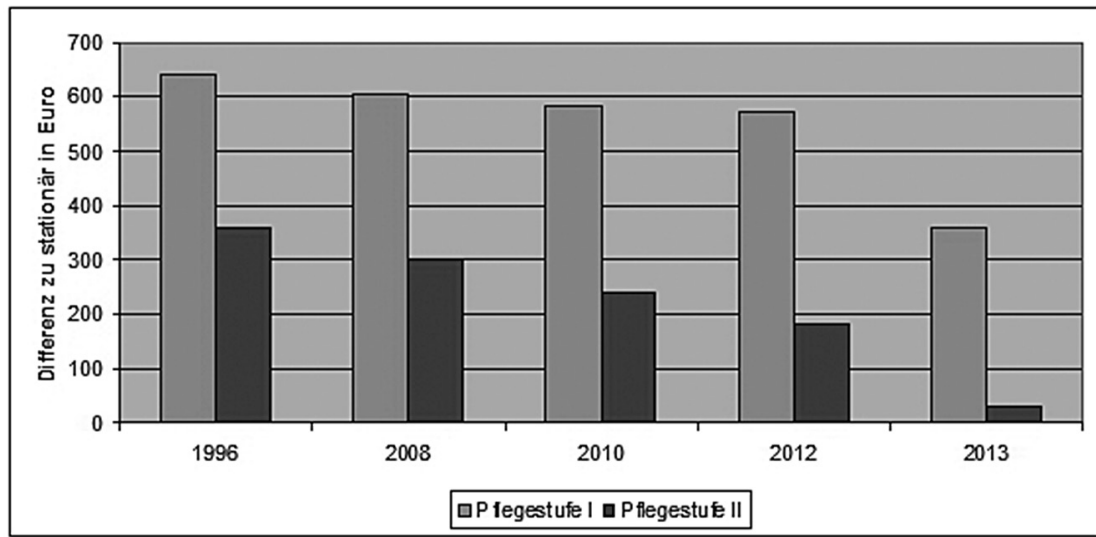

Abb. 1: Das Pflegeneuordnungsgesetz verringert die unterschiedlichen Sachleistungshöhen bei ambulant versorgten Personen mit "erheblich eingeschränkter Alltagskompetenz".

entwicklung notwendigen Ressourcen bereitstellte. Und mit erhöhter Frequenz etablierte Qualitätsprüfungen des Medizinischen Dienstes der Krankenversicherung (MDK) stellten neue, teils umstrittene Maßstäbe an den Pflegealltag dar, die zu implementierende Prozesse nach sich zogen.

Die Grenzen der zumutbaren, ohne zusätzliche Ressourcen umzusetzenden Veränderungen für die Pflegepraxis sind erreicht, wenn nicht überschritten. Die Verschnaufpause, die sich durch die aufgeschobene Umsetzung eines neuen Pflegebedürftigkeitsbegriffs ergibt, ist dringend nötig.

Die seit Start der Pflegeversicherung propagierte Maxime »ambulant vor stationär « wird im Pflegeneuordnungsgesetz ernsthaft angegangen durch Verringerung der unterschiedlichen Sachleistungshöhen für ambulante und stati-

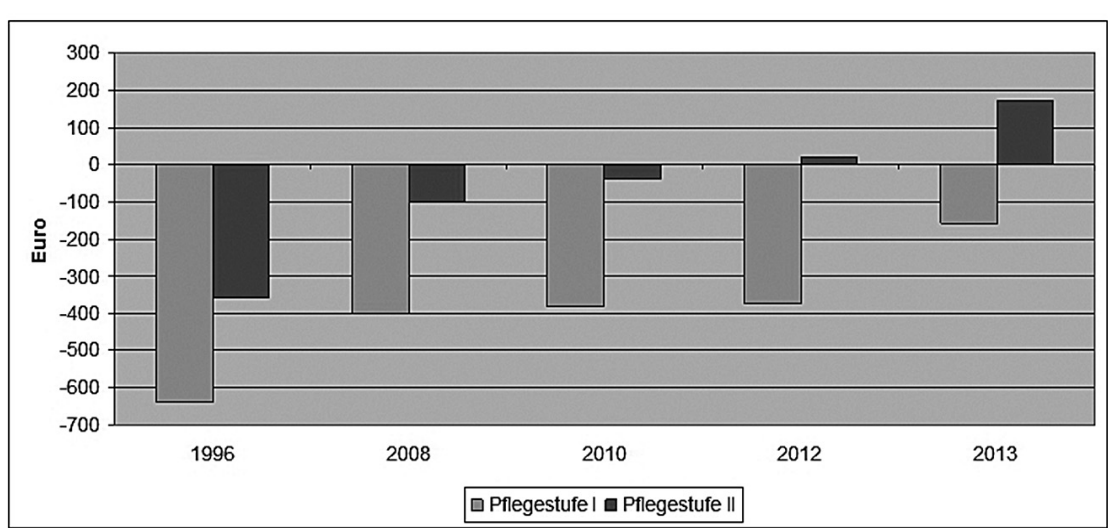

Abb. 2: Ambulant versorgte pflegebedürftige Menschen ohne kognitive Beeinträchtigungen erhalten weiterhin weniger als Pflegeheimbewohner. Allerdings können ambulant Versorgte mit Pflegestufe zahlreiche zusätzliche Leistungen in Anspruch nehmen.

onäre Leistungsempfänger. Diese schon durch das Pflegeweiterentwicklungsgesetz - die SGB XI-Reform zum 1. August 2008 - eingeleitete Entwicklung führt beim Pflegeneuordnungsgesetz in Pflegestufe 2 dazu, dass 97,7 Prozent des stationären Sachleistungsniveaus bei ambulant versorgten Personen mit »erheblich eingeschränkter Alltagskompetenz« gemäß $\ 45$ b SGB XI erreicht werden (vgl. Abb. 2). Vor den Reformen von 2008 und 2012 betrug die Differenz noch 359 Euro oder knapp 72 Prozent des stationären Sachleistungsbetrags in Pflegestufe 2. Ohne kognitive Beeinträchtigungen gemäß $\mathbb{4} 45 \mathrm{~b}$ SGB XI erhalten Pflegebedürftige in Pflegestufe 2 aber weiterhin nur 44 Prozent der stationären Sachleistungen oder 573 Euro weniger als Pflegeheimbewohner (vgl. Abb. 1). Bei Pflegestufe 1 bleibt ein größerer Abstand: Die ambulanten Sachleistungen sind 573 Euro geringer als bei Pflegeheimbewohnern; bei kognitiver Beeinträchtigung nach $\mathbb{S} 45 \mathrm{a}$ SGB XI beträgt der Unterschied noch 358 Euro.

Allerdings hinkt der Vergleich. Im Gegensatz zu Pflegeheimbewohnern haben alle ambulant Versorgten mit Pflegestufe zusätzlich zu den Sachleistungen auch Anspruch auf einen bunten Strauß weiterer Leistungen. Die Unterstützung für Kurzzeitpflege, Besuch einer Tagespflege und Monatsbeträge von 100 bzw. 200 Euro bei eingeschränkter Alltagskompetenz bleiben unverändert bestehen. Ambulanten Leistungserbringern bietet das Pflegeneuordnungsgesetz damit die Perspektive auf eher steigende Leistungsnachfrage in den beiden unte- 
ren Pflegestufen und durch Personen, die unterhalb der Pflegestufe erstmals finanzielle Unterstützung erhalten.

Die Annäherung der ambulanten und stationären Sachleistungen reduziert die Wahrscheinlichkeit finanziell motivierter Betreuungsentscheidungen: Nicht die Wohnform, sondern der Hilfebedarf bewirkt die Höhe der Versicherungsleistung.

Pflegeanbieter, die ihre Wohn- und Betreuungskonzepte weiterentwickeln wollen, müssen damit künftig weniger fragen, ob das ins Auge gefasste Angebot ambulant oder stationär sein soll. Eine stärkere Flexibilisierung der Wohnformen wird nun auch vom Gesetzgeber gewünscht. Die Erwartung an die Entwicklung neuer Wohnformen, die »bewohnerorientiert individuelle Versorgung anbieten ", und zwar »alternativ zu stationären Einrichtungen « findet sich im neu eingefügten $\mathbb{} \int 5 \mathrm{f}$ SGB XI. Für deren wissenschaftlich gestützte Weiterentwicklung werden zehn Millionen Euro zur Verfügung gestellt. Laut Gesetzesbegründung wird dabei nicht nur an Wohngemeinschaften gedacht, sondern auch an " ambulantisierte $<\mathrm{Be}$ treuungsformen, die bewohnerorientiert vor hochprofessionellem, institutionengestütztem Hintergrund modulhaft individuelle Versorgung anbieten, die bedarfsweise in Anspruch genommen werden kann«. Wünschenswert ist, dass Erkenntnisse dieser Erprobungen künftig für Pflegeheime allgemein die strukturellen Zwänge abbauen, zu denen sie gesetzlich verdammt sind.

Inhaltlich setzt das Pflegeneuordnungsgesetz neben Leistungen der Grundpflege und hauswirtschaftlichen Versorgung erstmals die "häuslichen Betreuung «. Es versteht diese drei Bereiche gemeinsam als »pflegerische $\mathrm{Be}$ treuungsmaßnahmen« (vgl. $\mathbb{S} 124$ Abs. 2 SGB XI).

Hintergrund ist das zunehmende Bewusstsein für die mit Demenz einhergehenden Alltagserfordernisse in Pflegehaushalten. Die Gesetzesbegründung spricht sogar von einem »Orientierungswechsel, was unter Pflege zu verstehen ist [...] und wie vor allem der besondere, in der Regel betreuerisch und weniger grundpflegerisch ausgerichtete Bedarf von Demenzkranken zu berücksichtigen ist «.

Dies kann als Reaktion auf das seit 1995 sehr eng verstandene Tätigkeits- spektrum der ambulanten Pflegedienste gewertet werden, das ohne gesetzlichen Zwang entstand: Die zwischen den Verbänden der Leistungserbringer und der Pflegekassen auf Landesebene verhandelten Gebührenordnungen haben Pflegesachleistungen ausschließlich als konkrete Verrichtungen formuliert - analog zum Verrichtungsbezug in der Definition der Pflegebedürftigkeit in $\mathbb{1 4}$ SGB XI. Entgelte für Zeiteinheiten, für die inhaltlich nichts Näheres bestimmt ist, waren nicht als Pflegesachleistung abrechenbar - eine Ausnahme gibt es im Bundesland Bremen.

Pflegehaushalten fehlte dadurch Flexibilität in der Beauftragung der Pflegedienste - was nicht auf der Gebührenordnung stand, wurde als nicht abrufbar verstanden. Lange Zeit fehlte auf den Preislisten ambulanter Dienste selbst ein Hinweis auf den Preis für eine Stunde oder eine Viertelstunde. Durchgängig kritisch wird in der Pra- gelegenheiten in Betracht. [...] Sonstige Hilfen schließen auch Hilfen mit ein, bei denen ein aktives Tun nicht im Vordergrund steht.« Dass bei solchen Aktivitäten nicht dieselbe pflegerische Expertise an die Leitungsebene gefordert werden muss wie bei körperbezogenen Pflegetätigkeiten, wird angenommen. Dies kann interessante Fachdiskurse zu Pflegeverständnis und benötigter Qualifikation auslösen.

\ 125 SGB XI ermöglicht die Zulassung eigenständiger Betreuungsdienste neben den klassischen Pflegediensten allerdings nur im Modellstatus. Für die Leistungsanbieter ergibt sich hier eine begrüßenswerte Option für Personalkonzepte, die flexibler auf individuelle Situationen ausrichtbar sind.

Neben den beschriebenen, eher strategisch relevanten gesetzlichen Änderungen enthält das Pflegeneuordnungsgesetz einige kleinere, operativ wirksame Verbesserungen. Positiv ist die

\section{"Die Mauern zwischen sstationären und sambulantens Angeboten werden niedriger"}

xis allerdings das Wahlrecht zwischen inhaltlich definierter Modulleistung und dem Einkauf einer Zeiteinheit eingeschätzt, das Nutzern ambulanter Dienste ab 2013 zwingend gewährt werden muss ( $\int 89$ Abs. 3 SGB XI). Die Praktikabilität der Gleichzeitigkeit von aufwandsabhängigen und aufwandsunabhängigen Entgelten ist fraglich und bleibt abzuwarten.

Als häusliche Betreuung werden gemäß $\int 124$ Abs. 2 »Aktivitäten im häuslichen Umfeld « verstanden, »die dem Zweck der Kommunikation und der Aufrechterhaltung sozialer Kontakte dienen « sowie der »Unterstützung bei der Gestaltung des häuslichen Alltags «. In der Begründung zum Gesetzentwurf heißt es dazu: "Spaziergänge in der näheren Umgebung können ebenso zur Aufrechterhaltung sozialer Kontakte beitragen wie die Ermöglichung des Besuchs von Verwandten und Bekannten oder die Begleitung zum Friedhof. Darüber hinaus kommen Unterstützungsleistungen bei der Regelung von finanziellen und administrativen An-
Erkenntnis des Gesetzgebers, dass die in Pflegehaushalten meist von Angehörigen übernommene Schlüsselaufgabe, die Versorgung zu koordinieren, auch in ambulant betreuten Wohngemeinschaften anfällt. Dafür können sich Bewohner einer Wohngemeinschaft monatlich 200 Euro von der Pflegekasse erstatten lassen (vgl. \38a SGB XI). Der Bundesgesetzgeber tut sich offensichtlich weniger schwer mit dieser Wohnform als manche Landesgesetzgeber, die das Ordnungsrecht für Wohngemeinschaften gestalten.

Die zum 1. Juli 2008 eingeführten, von den Pflegekassen finanzierten zusätzlichen Betreuungskräfte nach \ 87b SGB XI im Pflegeheim waren ein Erfolg. Ab 2013 wird auch Tagespflegeeinrichtungen der Einsatz solcher zusätzlichen Betreuungskräfte ermöglicht. Der Gesetzgeber hat dabei realistisch gesehen, dass sich in der Tagespflege mit viel geringeren Platzzahlen als im Pflegeheim beim gesetzlich geregelten Personalschlüssel von 1:24 keine hohen Beschäftigungsumfänge 
ergeben können. Daher dürfen Tagespflegen die Betreuungsassistenzen auch auf geringfügiger Basis beschäftigen. So können die ohnehin wenigen, regulär in der Tagspflege eingesetzten Kräfte zumindest stundenweise entlastet werden. Und für die anspruchsberechtigten Gäste ist es eine echte Leistungsverbesserung.

Erstmals seit zehn Jahren enthält eine SGB XI-Reform keine wesentliche Verschärfung der Qualitätsanforderungen und Prüfung der Normkonformität von Einrichtungen und Diensten. Dagegen zielt sie auf Unterstützung der Pflegeheime, die sich um eine gute Zusammenarbeit mit den Haus- und Fachärzten zum Wohle der Bewohner bemühen, diese aber nicht selbst sicherstellen können. Artikel 2 des Pflegeneuordnungsgesetz enthält Änderungen im Krankenversicherungsrecht, von denen drei die ärztliche Versorgung im Pflegeheim betreffen. Das dortige Alltagsproblem, dass eine Dekolonisation von Trägern des Methicillin-resistenten Staphylococcus aureus (MRSA) durch Hausärzte an fehlenden Positionen in der Gebührenordnung der Ärzte scheitert, wird ebenso angegangen $(\mathbb{S} 92$ Abs. 7 SGB V) wie die unzureichende Bereitschaft von Fachärzten zu Hausbesuchen
( $\$$ 87a SGB V). Speziell für Menschen mit erheblich eingeschränkter Alltagskompetenz, die Zahnarztpraxen nicht aufsuchen können, wird eine zusätzliche Vergütung in der zahnärztliche Gebührenordnung (EBM) für aufsuchende zahnärztliche Behandlung vorgegeben ( $\$ 87$ Abs. 2j SGB V). Flankierend werden die Verbände auf Bundesebene bis zum 30. September 2013 verpflichtet, »Anforderungen an eine kooperative und koordinierte ärztliche und pflegerischen Versorgung « von Pflegeheimbewohnern zu verabschieden $(\mathbb{S} 119 \mathrm{~b}$ SGB V).

Ein trauriges Kapitel in der Umsetzung der Pflegeversicherung ist die jahrelange Ignoranz der Pflegekassen und Sozialhilfeträger, was die Berücksichtigung tarifbedingter Personalkosten in Entgelten betrifft. Der seit 2010 für die Pflege geltende Mindestlohn ließ nach Erfahrungen der Vergangenheit befürchten, dass dieser zum Maßstab für Entgeltverhandlungen werden könnte. In $\mathbb{S} 84$ und 89 SGB XI fügt das Pflegeneuordnungsgesetz deshalb den Hinweis ein, dass stationäre und ambulante Pflegeentgelte bei wirtschaftlicher Betriebsführung die Personalaufwendungen finanzieren können müssen. In der Gesetzesbegründung wird »die Zahlung von Tariflöhnen ausdrücklich als wirtschaftliche Betriebsführung bezeichnet [...] Um auch die Kostenträger auf die Richtigkeit dieser Vorgehensweise hinzuweisen und unnötige Sozialgerichtsverfahren gegen ablehnende Entscheidungen der Kostenträger zu vermeiden, erfolgt eine entsprechende Klarstellung." Ob diese unmissverständliche Vorgabe von Pflegekassen und Sozialhilfeträgern tatsächlich durchgängig beachtet wird, bleibt abzuwarten.

Es ist diese Erfahrung der Vergangenheit, die Skepsis weckt bei der Erwartung, mit dem neuen Pflegebedürftigkeitsbegriff würde alles besser. Demenzkranke Leistungsempfänger mögen dadurch mehr Gerechtigkeit erfahren. Doch für Pflegeanbieter ist essentiell, wie sich die mit Pflegekassen und Sozialhilfeträger ausgehandelten Konditionen gestalten. Damit sich diese verbessern, bedarf es keiner anderen Zugangsschwelle zu Pflegekassenleistungen, sondern einer aufwandsorientierten Entgeltgestaltung und einer neuen Verhandlungskultur.

\section{Literatur zur Pflegereform}

Am 1. Januar 2013 traten die letzten Neuregelungen des neuen Pflege-Neuausrichtungs-Gesetz in Kraft. Dieses Gesetz ist seit vielen Jahren die umfänglichste Reform im Bereich der Pflegeversicherung nach SBG XI und führt zu erheblichen Änderungen im Leistungs- und Leistungserbringungsbereich der Pflegeversicherung. Die 4. Auflage des Lehr- und Praxiskommentars zum SGB XI bringt Interpretationssicherheit in den neuen Rechtsfragen. Utz Krahmer, Markus Plantholz, Thomas Klie (Hg.). Sozialgesetzbuch XI. Soziale Pflegeversicherung. 4. Auflage. Nomos Verlagsgesellschaft, Baden-Baden 2013. Ca. 1.300 Seiten. 89,- Euro. ISBN 978-38329-5042-2.

Das Handbuch zum Pflegesozialrecht erscheint als Standardwerk bereits in der 5. Auflage. Es umfasst ebenfalls die Neuerungen des Pflege-NeuausrichtungsGesetzes, berücksichtigt alle Änderungen bei einer Vielzahl von Pflegesozialleistungen.
Heinrich Griep, Heribert Renn (Hg.): Pflegesozialrecht. Ein Handbuch. 5. Auflage. Nomos Verlagsgesellschaft, Baden-Baden 2013. 267 Seiten. 39,- Euro. ISBN 978-38329-7793-1.

Die neue Textsammlung zum Pflegerecht mit einer Einführung von Prof. Dr. Thomas Klie bietet alle Rechtsregeln für Pflegeberufe auf einen Blick. Eine alphabetische Gliederung, eine systematische Inhaltsübersicht und ein ausführliches Stichwortverzeichnis gestatten einen schnellen Zugang zu den gesuchten Regelungen.

Thomas Klie, Ulrich Stascheit (Hg.): Gesetze für Pflegeberufe. Textsammlung. 13. Auflage. Nomos Verlagsgesellschaft, Baden-Baden 2013. 2.086 Seiten. 24,--Euro. ISBN 978-3-8329-7963-8.

Der neue Großkommentar zum Sozialrecht bietet Orientierung an den wesentlichen Grundstrukturen der auch für die Pflege einschlägigen Gesetze wie SGB $\vee$ und SGB XI und stellt die Leistungsansprüche in den Mittelpunkt. Die Kommentierung beschränkt sich nicht auf die Wiedergabe der einschlägigen Rechtsprechung, sondern geht weiter, indem bisher ungelöste Probleme identifiziert und Wege zu ihrer Lösung aufgezeigt werden.

Josef Berchtold, Stefan Huster, Martin Rehborn (Hg.). SGB V und SGB XI. Großkommentar. Nomos Verlagsgesellschaft, Baden-Baden 2013. Ca. 2.500 Seiten. 198,Euro. ISBN 978-3-8329-6161-9.

Die Zeit ist reif für grundlegende Änderungen im Gesundheitswesen. Das Buch "Masterplan Gesundheitswesen 2020" analysiert in klaren Worten den Status quo und zeigt Wege zu einem zukunftsfesten Gesundheitswesen auf. Die Herausgeber fordern eine gesamtgesellschaftliche Kraftanstrengung spätestens nach den nächsten Bundestagswahlen.

Bodo Klein, Michael Weller (Hg.): Masterplan Gesundheitswesen 2020. Nomos Verlagsgesellschaft, Baden-Baden 2012. 225 Seiten. 39,- Euro. ISBN 978-3-83296875-5. 